\title{
Adenoma velloso de esófago en la mucosa ectópica gástrica: reporte de un caso
}

\section{Villous adenoma of the esophagus in ectopic gastric mucosa: A case report}

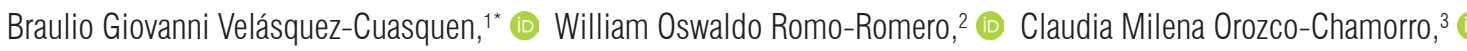
Angela Gicel Molano-Narváez, ${ }^{4}$ [D Andrés Ordóñez-Ruiz. ${ }^{5}$

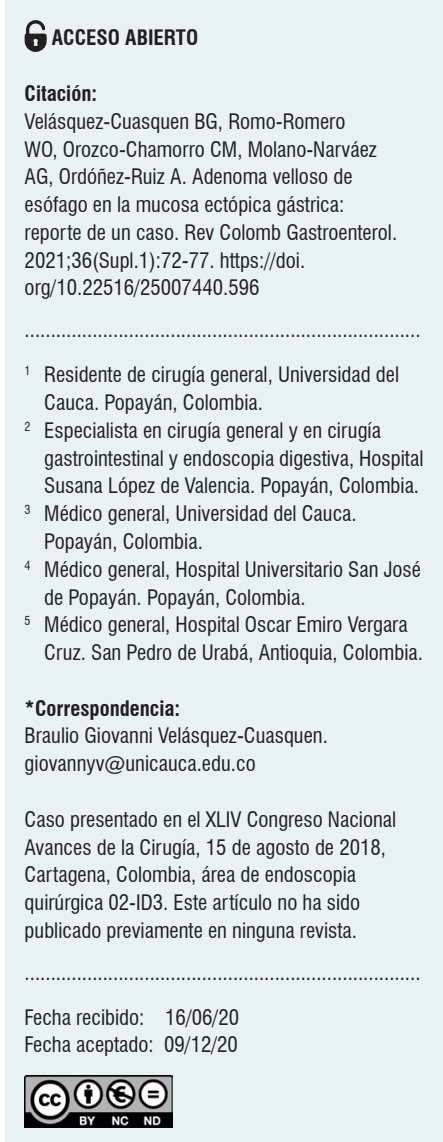

\begin{abstract}
Resumen
Introducción: los adenomas vellosos son lesiones del tubo digestivo con tendencia alta a la malignidad. Su ubicación en parches de mucosa gástrica ectópica en el esófago cervical determina una presentación atípica de interés tanto clínico como patológico. Objetivo: presentar un caso de adenoma velloso en la mucosa gástrica ectópica del esófago cervical. Métodos: describir el caso de un paciente con diagnóstico de adenoma velloso y realizar una revisión de la literatura disponible hasta el momento. Resultados: se identificó mediante un estudio histopatológico un adenoma tubulovelloso con displasia de bajo grado, cuya ubicación endoscópica era un parche de mucosa ectópica gástrica en el esófago cervical. Conclusiones: la ubicación de los adenomas vellosos en el esófago cervical puede predisponer al desarrollo de lesiones neoplásicas. La evaluación detallada de este segmento, con técnicas como la cromoendoscopia digital de alta definición, permiten la detección temprana de estas lesiones y su oportuna intervención.
\end{abstract}

\section{Palabras clave}

Adenoma velloso, disección endoscópica de la submucosa (DES), mucosectomía endoscópica, reflujo gastroesofágico.

\section{Abstract}

Introduction: Villous adenomas are lesions of the digestive tract with a high tendency to malignancy. Its location in ectopic gastric mucosa patches in the cervical esophagus is an atypical presentation of clinical and pathological interest. Objective: To present a case of villous adenoma in ectopic gastric mucosa of the cervical esophagus. Methods: A case study of a patient with a diagnosis of villous adenoma is presented, as well as a review of the current literature. Results: A tubulovillous adenoma with low-grade dysplasia was identified by histopathological study. Its endoscopic location was a gastric ectopic mucosa patch in the cervical esophagus. Conclusions: The location of villous adenomas in the cervical esophagus may predispose to the development of neoplastic lesions. Detailed evaluation of this segment using techniques, such as highdefinition digital chromoendoscopy, would allow for early detection and treatment of these lesions.

\section{Keywords}

Villous adenoma; Endoscopic submucosal dissection (ESD); Endoscopic mucosectomy; Gastroesophageal reflux.

\section{INTRODUCCIÓN}

Los adenomas vellosos se han descrito como lesiones polipoides, preneoplásicas, que representan del $5 \%$ al $15 \%$ de los pólipos adenomatosos; exhiben una arquitectura papilar y vellositaria con alta tendencia a la displasia en relación con una alta actividad mitótica y renovación epitelial, con tasas de transformación maligna hasta de $72 \%$. Su localiza- 
ción es frecuente a nivel colorrectal, y son pocos los casos reportados en el tracto digestivo superior, especialmente en esófago $(1,2)$. El hallazgo de este tipo de lesiones en un parche de mucosa ectópica gástrica en el esófago cervical se ha descrito en pocos casos, los cuales frecuentemente son subdiagnosticados, pues se localizan en la zona de paso rápido y menor evaluación con el endoscopio; además, son considerados de origen congénito; por tanto, de bajo potencial para malignidad $(2,3)$.

Se presenta un caso de adenoma velloso asentado en un parche de mucosa gástrica ectópica localizado en el esófago cervical. Se realizó una revisión de la literatura en relación con esta rara entidad (1-3).

\section{CASO CLÍNICO}

Se trata de un paciente masculino de 41 años con antecedentes familiares de cáncer gástrico materno y antecedentes personales de hemorroidectomía y gastritis crónica. 2 años antes de la consulta recibió tratamiento para Helicobacter pylori. El paciente consultó por un cuadro clínico de 6 meses de evolución, consistente en epigastralgia y pirosis intensa de predominio nocturno. El paciente niega episodios de emesis o sangrado digestivo. Como estudios previos, aportó una gammagrafía de esófago que evidenció al menos 10 episodios de reflujo gastroesofágico, el más significativo fue de 10 segundos, que se extendió hasta el tercio proximal del esófago. Durante la endoscopia de luz blanca, en el esófago cervical e inmediatamente por debajo del esfínter esofágico superior se observaron 2 islas de mucosa similar a la del antro gástrico, de color salmón y con bordes bien definidos; la isla mayor era de unos $18 \times 26 \mathrm{~mm}$. En la evaluación con luz de banda estrecha (narrow band imaging [NBI]), estas 2 islas de mucosa columnar se observaron nítidamente y, además, en el extremo superior de la isla mayor se observaron 2 pequeños parches de mucosa blanquecina, ligeramente levantada de aspecto adenomatoso. La cavidad gástrica presentaba una erosión aislada prepilórica y gastropatía crónica con atrofia multifocal (Figura 1).

El reporte histopatológico informó gastritis crónica atrófica, $H$. pylori positivo, esofagitis y lesión sugestiva de adenoma velloso. Se indicó un tratamiento triconjugado de primera línea para $H$. pylori.

Se programó la resección de la lesión mediante una técnica de disección endoscópica de la submucosa (DES). Durante el procedimiento, posiblemente por el proceso de cicatrización secundaria a las biopsias realizadas, no se logró elevar satisfactoriamente la lesión mediante la inyección submucosa de solución salina con azul índigo carmín; por esta razón, se decidió cambiar la técnica de resección y se realizó la resección completa con el kit de mucosectomía endoscópica de Olympus, EMR Kit Modelo K-009 (inyector, cap angulada y asa de polipectomía) y con ayuda de la unidad electroquirúrgica ERBE VIO $200 \mathrm{~S}$ (modo endocut $\mathrm{Q}$ efecto nivel 3, duración de corte 1 y con intervalo de corte de 6) (Figura 2). Finalmente, el parche de mucosa gástrica ectópica fue completamente resecada en 4 fragmentos, incluidos los focos de tejido adenomatoso. No se observó tejido columnar residual y no se realizó ablación de los bordes de resección debido a que no hubo sangrado
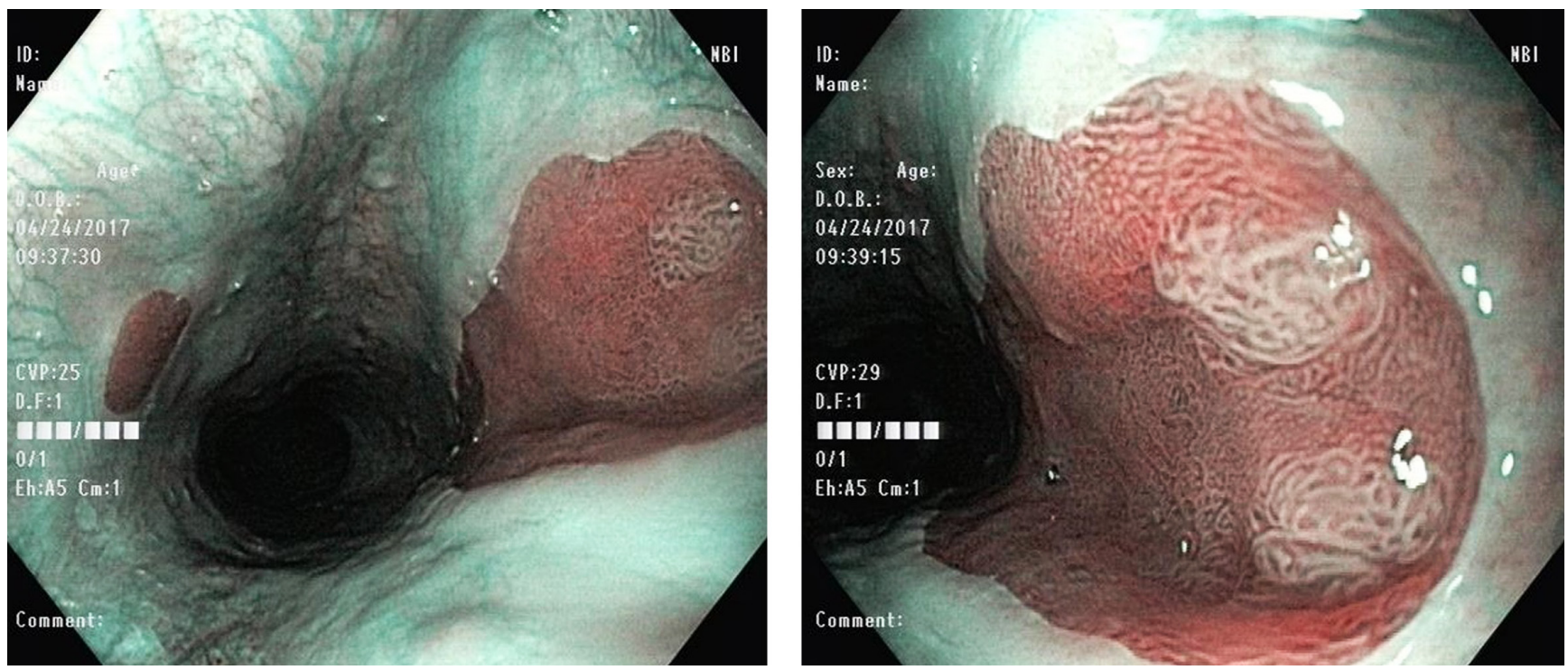

Figura 1. Parche de mucosa gástrica ectópica en el esófago cervical, con 2 islas de tejido de aspecto adenomatoso (NBI). 
y en consideración a que la mucosa columnar por sí misma no corresponde a una lesión premaligna (Figura 3).

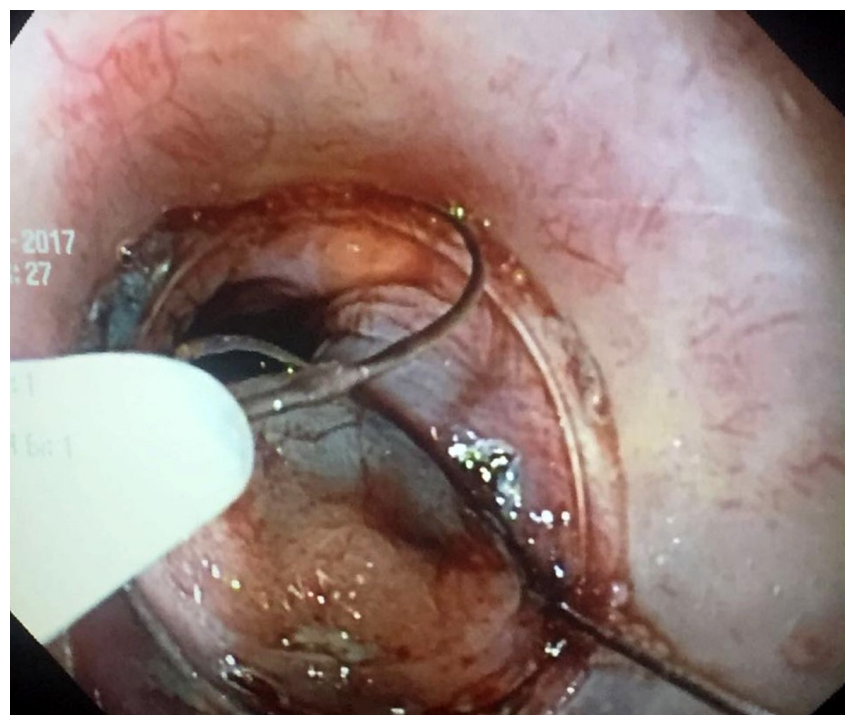

Figura 2. Mucosectomía con EMR kit.

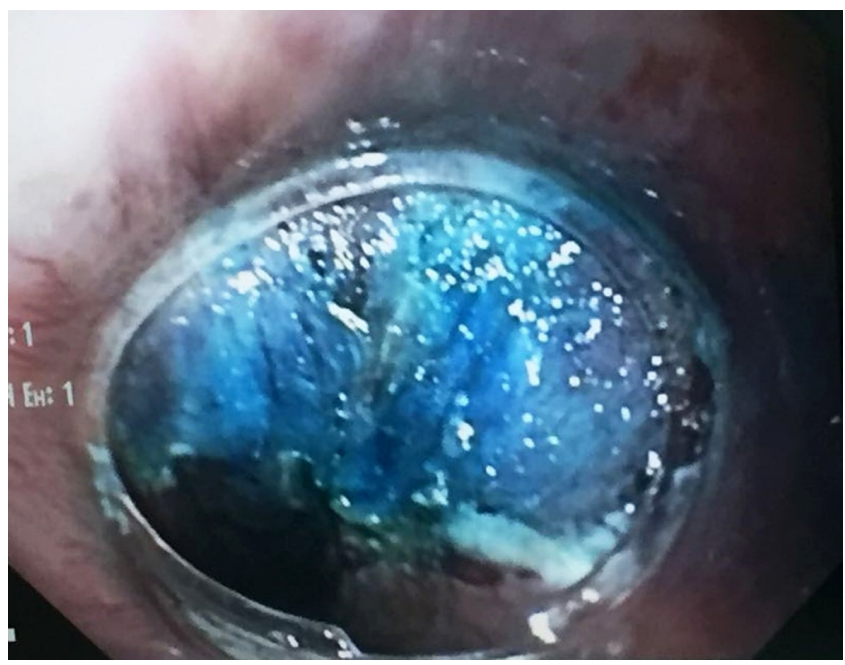

Figura 3. Imagen endoscópica posmucosectomía. Lesión completamente resecada.

Los fragmentos resecados fueron enviados para estudio histopatológico. Los más grandes extendidos en una pieza de corcho y fijados con alfileres.

El estudio histopatológico reportó una mucosa esofágica comprometida por una lesión displásica, conformada por glándulas tubulares y vellosas, revestida por un epitelio cilíndrico simple, núcleos hipercromáticos sin evidencia de pérdida de la polaridad ni compromiso de la membrana basal. En la lámina propia se observó un infiltrado inflamatorio crónico en escasa cantidad, compatible con adenoma velloso, con displasia de bajo grado (Figuras 4 y 5 ).

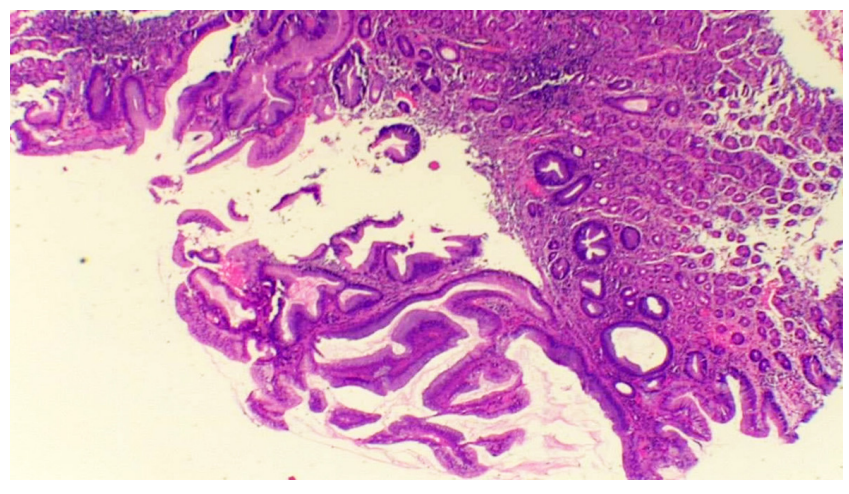

Figura 4. Imagen microscópica. Tinción hematoxilina-eosina: aumento de $10 \mathrm{x}$. Hallazgos compatibles con adenoma velloso.

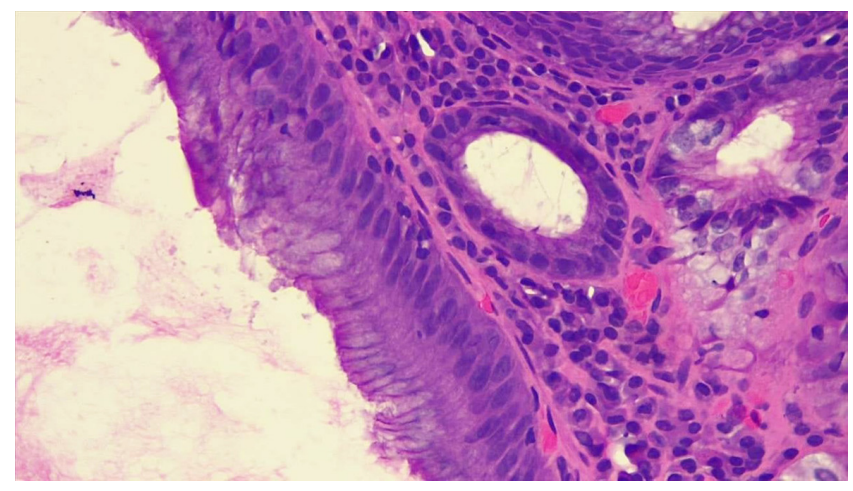

Figura 5. Imagen microscópica. Tinción hematoxilina-eosina, aumento de $40 \mathrm{x}$. Hallazgos compatibles con adenoma velloso.

El paciente presentó una evolución clínica satisfactoria, sin manifestar síntomas gastrointestinales ni sangrado posquirúrgico. Se realizó un nuevo control endoscópico 6 meses después de la resección de la lesión, en el que se evidenció el esófago con tejido blanquecino asociado con reepitelización, una pequeña cicatriz central en espejo y una pequeña isla de mucosa columnar de $5 \mathrm{~mm}$. El resto del estudio endoscópico fue normal (Figura 6).

El paciente continúa en control por el grupo de gastroenterología quirúrgica con una adecuada evolución clínica.

\section{DISCUSIÓN}

Los pólipos neoplásicos, también denominados adenomas o pólipos adenomatosos, son lesiones premalignas con capacidad para transformarse en adenocarcinomas. Se clasifican en adenoma tubular, tubulovelloso y velloso, cuya característica histológica es un epitelio displásico. A su vez, se subdividen en lesiones de bajo y alto grado de acuerdo con el tipo de displasia, al nivel de aglomeración nuclear e hipercromasia, según su actividad mitótica, diferenciación citoplasmática y distorsión de la arquitectura celular. Los 

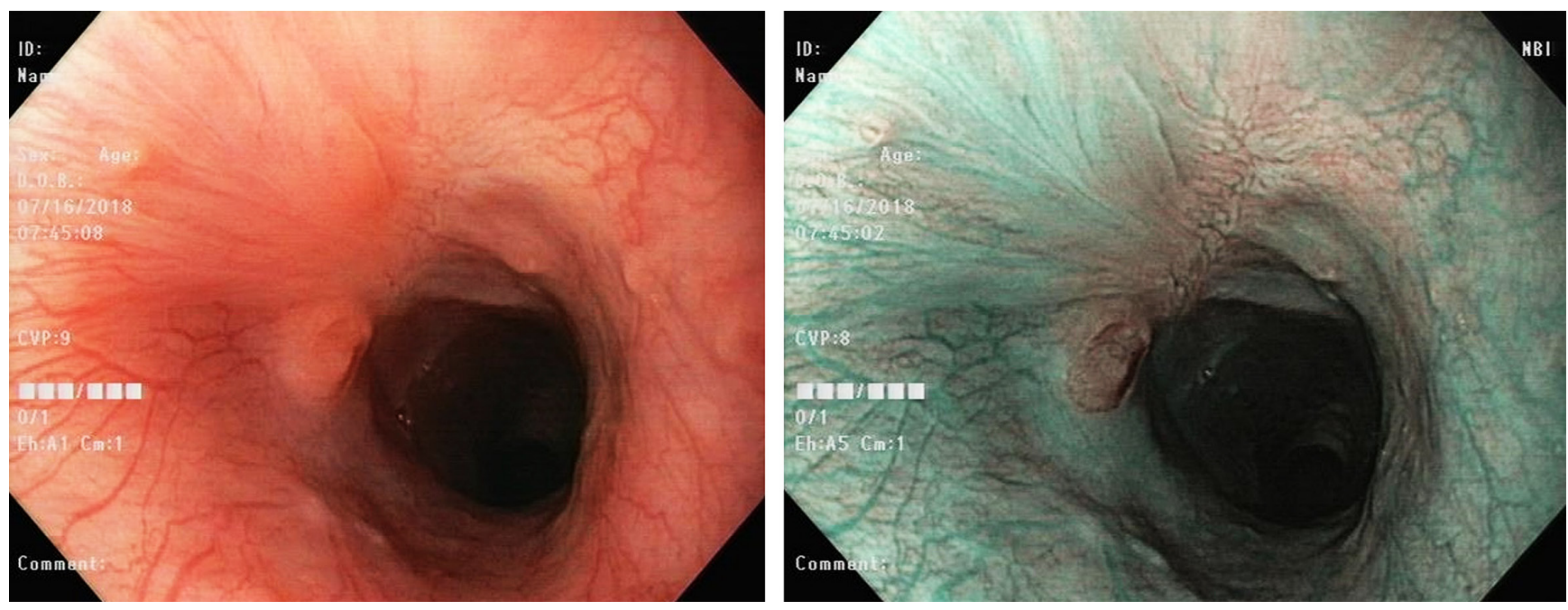

Figura 6. Imágenes endoscópicas de control, resección de adenoma velloso.

adenomas tubulares presentan entre $0 \%$ y $25 \%$ de histología vellosa; el adenoma tubulovelloso, entre $20 \%$ y $75 \%$; y el adenoma velloso, entre $75 \%$ y $100 \%$ (1-4).

Los adenomas vellosos generalmente poseen arquitectura papilar vellositaria, que se relaciona más con lesiones $>$ $2 \mathrm{~cm}$. Este tipo de pólipo se presenta en hombres y mujeres, sobre todo en la sexta y séptima década de la vida. Se caracterizan por ser estructuras glandulares largas que se extienden desde la superficie hasta el centro del pólipo. Este subtipo se asocia con adenomas de mayor tamaño, grados más graves de displasia y mayor tendencia a la malignidad, y se describen tasas de alrededor del $72 \%$ de transformación maligna (4-6). Representan del $5 \%$ al $15 \%$ de todos los adenomas y se presentan con mayor frecuencia en el recto $y$ recto sigmoide, y escasamente se han reportado en otras partes del tracto digestivo $(1,2,4)$.

Esta condición probablemente está subdiagnosticada y la significancia clínica no es del todo clara, dado que un porcentaje importante de pacientes no refiere síntomas asociados. No obstante, a pesar de que los hallazgos endoscópicos pueden sugerir un potencial neoplásico, es el estudio histopatológico el que determina el grado de malignidad de dicha lesión (1). Los adenomas vellosos gástricos se presentan como lesiones asintomáticas en más del $90 \%$ de casos, aunque pueden presentar manifestaciones como sangrado, anemia, dolor abdominal o síndrome pilórico. Pese a que la localización gástrica más frecuente es en el antro gástrico, series como las presentadas por Miller y colaboradores muestran que pueden presentarse en la unión gastroesofágica $(4,5,7)$.

En el caso que exponemos, se identificó la presencia de 2 lesiones endoscópicamente compatibles con neoformacio- nes adenomatosas asentadas sobre una isla de mucosa gástrica ectópica en el esófago cervical. Estas áreas de mucosa ectópica gástrica son hallazgos relativamente frecuentes; no obstante, la descripción de prevalencia varía ampliamente en la literatura, con datos entre $0,18 \%$ y $1,6 \%$. La discrepancia puede relacionarse con que frecuentemente estas zonas son pasadas por alto o ignoradas durante el estudio endoscópico $(10,11,13)$. Estas islas de mucosa gástrica ectópica se han asociado con un origen congénito y con baja tendencia intrínseca a desarrollar malignidad. A pesar de esto, desde 1950 hasta el 2016 se han reportado más de 50 casos de adenocarcinomas que crecen a partir de parches de mucosa gástrica ectópica $(8,9,11,16)$.

Tomohiro Kadota y colaboradores encontraron un total de 27 casos de adenocarcinomas esofágicos formados a partir de islas de mucosa ectópica gástrica en pacientes con edades entre los 43 y los 83 años, y la disfagia fue el principal síntoma $(11,12)$. En el $96 \%$ de casos, la localización de la lesión neoplásica fue en el esófago cervical o esófago torácico superior, lugar de localización predilecta de las islas de mucosa ectópica gástrica. Los principales subtipos histopatológicos fueron adenocarcinomas bien diferenciados y moderadamente diferenciados, y se reconoció tejido ectópico gástrico adyacente a la lesión en todos los casos (13-16).

Los parches de mucosa gástrica ectópica no son procesos adaptativos del tejido esofágico, sino que corresponden a regiones de mucosa gástrica secuestrados en el esófago después de un proceso de reemplazo embriológico de un epitelio columnar por el epitelio esofágico escamoso estratificado. No obstante, la presencia de metaplasia intestinal, descrita entre el $0 \%$ y $12 \%$ de los casos, indicaría que estas 
zonas de mucosa gástrica de igual forma pueden sufrir procesos de transformación con determinados estímulos deletéreos y también pueden llegar a representar condiciones preneoplásicas (8-15).

Se han planteado distintas teorías para explicar la secuencia de cambios fisiopatológicos que conducen de una isla de mucosa ectópica gástrica hacia un adenocarcinoma esofágico, como la secuencia metaplasia-displasia-adenocarcinoma, y también se ha asociado la presencia de H. pylori con predisposición a transformación maligna. Sin embargo, bajas tasas de malignidad en estas lesiones en comparación con entidades como esófago de Barret han llevado a sugerir que las islas de mucosa ectópica gástrica no son per se lesiones premalignas $(8-10,16,17)$.

Este caso, que es uno de los primeros reportados de este tipo de entidad en Colombia, ayuda a reflexionar que el riesgo de desarrollo de lesiones con potencial maligno en islas de mucosa gástrica ectópica clásicamente descritas como de baja tendencia a la malignidad, aunque son raras, pueden presentarse.

\section{CONCLUSIONES}

En pacientes sometidos a estudios endoscópicos, principalmente aquellos con enfermedad por reflujo gastroesofágico, se sugiere realizar una cuidadosa evaluación de la mucosa esofágica, especialmente del esófago cervical, en busca de la presencia de estos parches de mucosa gástrica ectópica. Es de suma importancia recordar que uno de los indicadores específicos de calidad de la esofagogastroduodenoscopia es la realización del examen completo de cada una de las estructuras (esófago, estómago y segunda porción del duodeno, incluida la retroflexión del estómago) que, además, debe estar claramente documentado en el informe final (18).

Idealmente, esta zona debe evaluarse mediante la técnica de imagen de banda estrecha (NBI), la cual se basa en la modificación del ancho de banda de la luz emitida, lo que permite conseguir información adicional sobre la mucosa y la morfología de los vasos superficiales. De esta manera, es útil en la demostración de áreas sospechosas de neoplasia en el epitelio escamoso del esófago (19).

El estudio endoscópico cuidadoso de heterotopías gástricas esofágicas está indicado para la valoración de hallazgos que sugieran premalignidad. La identificación de irregularidad en la mucosa debe ser indicación para la toma de biopsia y estudios histopatológicos complementarios. La identificación de adenomas en la mucosa esofágica debe indicarse para su resección endoscópica; su porcentaje de histología vellositaria va a tener relación con su potencial de malignidad.

\section{PARA LLEVAR A CASA}

En pacientes programados para endoscopia digestiva alta y especialmente aquellos que presenten algún tipo de sintomatología esofágica alta como disfagia, globo faríngeo o síntomas de reflujo gastroesofágico, se sugiere realizar una lenta y cuidadosa evaluación de la mucosa del esófago cervical y, de ser posible, utilizar cualquier técnica de cromoendoscopia digital para facilitar la identificación de los parches de mucosa gástrica ectópica.

\section{Fuente de financiación}

Se declara que no existieron apoyos financieros para la realización de este manuscrito.

\section{REFERENCIAS}

1. Delgado EM, Morro PI, de Oporto PM. Hipopotasemia por adenoma velloso. Síndrome de Mckittrick y Wheelock. FMC-Formación Médica Continuada en Atención Primaria. 2009;16(2):79-84. https://doi.org/10.1016/S1134-2072(09)70286-5

2. Wong WM, Shek TH, Chan CK, Lai KC. Gastrointestinal: Villous adenoma of the esophagus. J Gastroenterol Hepatol. 2004;19(10):1213. https://doi.org/10.1111/j.1440-1746.2004.03620.x

3. Strum WB. Colorectal adenomas. N Engl J Med. 2016;374(11):1065-1075. https://doi.org/10.1056/NEJMra1513581
4. Castro DJ, Marcano J, Peraza S, Ramírez L. Adenoma velloso de estómago: presentación de un caso. Gen. 2016;70(3):86-88.

5. Shaib YH, Rugge M, Graham DY, Genta RM. Management of gastric polyps: an endoscopy-based approach. Clin Gastroenterol Hepatol. 2013;11(11):1374-1384. https://doi.org/10.1016/j.cgh.2013.03.019

6. Jang CR, Choi SR, Cho JH, Koo YH, Han SH, Ryu SH, Lee DH, Jang JS, Lee JH, Roh MH, Cho JH. A case of giant gastric villous tumor with carcinomatous change. Korean J Gastroenterol. 2005;45(6):431-5.

7. Miller JH, Gisvold JJ, Weiland LH, McIlrath DC. Upper gastrointestinal tract: villous tumors. AJR Am J 
Roentgenol. 1980;134(5):933-6.

https://doi.org/10.2214/ajr.134.5.933

8. Mion F, Lambert R, Partensky C, Cherkaoui M, Berger F. High-grade dysplasia in an adenoma of the upper esophagus developing on heterotopic gastric mucosa. Endoscopy. 1996;28(7):633-5. https://doi.org/10.1055/s-2007-1005561

9. Jabbari M, Goresky CA, Lough J, Yaffe C, Daly D, Côté C. The inlet patch: heterotopic gastric mucosa in the upper esophagus. Gastroenterology. 1985;89(2):352-6. https://doi.org/10.1016/0016-5085(85)90336-1

10. Kaneko E, Kohda A, Honda N, Kino I. Incomplete tubular duplication of esophagus with heterotopic gastric mucosa. Dig Dis Sci. 1989;34(6):948-951. https://doi.org/10.1007/BF01540284

11. Peitz U, Vieth M, Evert M, Arand J, Roessner A, Malfertheiner P. The prevalence of gastric heterotopia of the proximal esophagus is underestimated, but preneoplasia is rare-correlation with Barrett's esophagus. BMC Gastroenterol. 2017;17(1):87. https://doi.org/10.1186/s12876-017-0644-3

12. Akbayir N, Alkim C, Erdem L, Sökmen HM, Sungun A, Basak T, Turgut S, Mungan Z. Heterotopic gastric mucosa in the cervical esophagus (inlet patch): endoscopic prevalence, histological and clinical characteristics. J Gastroenterol Hepatol. 2004;19(8):891-6. https://doi.org/10.1111/j.1440-1746.2004.03474.x

13. Azar C, Jamali F, Tamim H, Abdul-Baki H, Soweid A. Prevalence of endoscopically identified heterotopic gastric mucosa in the proximal esophagus: endoscopist dependent? J Clin Gastroenterol. 2007;41(5):468-71. https://doi.org/10.1097/01.mcg.0000225519.59030.8d
14. Poyrazoglu OK, Bahcecioglu IH, Dagli AF, Ataseven H, Celebi S, Yalniz M. Heterotopic gastric mucosa (inlet patch): endoscopic prevalence, histopathological, demographical and clinical characteristics. Int J Clin Pract. 2009;63(2):287-91. https://doi.org/10.1111/j.1742-1241.2006.01215.x

15. Gutierrez O, Akamatsu T, Cardona H, Graham DY, El-Zimaity HM. Helicobacter pylori and hetertopic gastric mucosa in the upper esophagus (the inlet patch). Am J Gastroenterol. 2003;98(6):1266-70. https://doi.org/10.1111/j.1572-0241.2003.07488.x

16. Kadota T, Fujii S, Oono Y, Imajoh M, Yano T, Kaneko K. Adenocarcinoma arising from heterotopic gastric mucosa in the cervical esophagus and upper thoracic esophagus: two case reports and literature review. Expert Rev Gastroenterol Hepatol. 2016;10(3):405-14. https://doi.org/10.1586/17474124.2016.1125780

17. von Rahden BH, Stein HJ, Becker K, Liebermann-Meffert $\mathrm{D}$, Siewert JR. Heterotopic gastric mucosa of the esophagus: literature-review and proposal of a clinicopathologic classification Am J Gastroenterol. 2004;99(3):543-51. https://doi.org/10.1111/j.1572-0241.2004.04082.x

18. López-Picazo Ferrer J, Alberca de las Parras F, Sánchez del Río A, Pérez Romero S, León Molina J, Júdez Gutiérrez F. Indicadores de calidad en endoscopia digestiva: introducción a los indicadores comunes de estructura, proceso y resultado. Rev Esp Enferm Dig. 2017;109(6):435-50. http://doi.org/10.17235/reed.2017.5035/2017

19. Tchekmedyian AJ, Pellisé M, Sáenz R. Imágenes de banda estrecha o Narrow band imaging (NBI): una nueva era en endoscopía digestiva. Rev Méd Urug. 2008;24(1):42-9. 